\title{
PERDA INSUFICIENTE DE PESO E OU AUSÊNCIA DE REMISSÃO DA DIABETE MELTO TIPO 2 APÓS A DERIVAÇÃO GÁSTRICA EM Y-DE-ROUX: FATORES QUE PODEM INFLUENCIAR OS RESULTADOS INSATISFATÓRIOS
}

\author{
Insufficient loss of weight and/or absence of DM2 remission after Roux-en-Y gastric bypass: \\ factors that may influence the unsatisfactory results
}

\author{
Anna Christina Charbel COSTA, Mariana Camara Martins Bezerra FURTADO, Eudes Paiva de GODOY, \\ Elenir Rose Jardim Cury PONTES, João Ricardo Filgueiras TOGNINI, Maria Lúcia IVO
}

Trabalho realizado no Serviço de Cirurgia da Obesidade e Doenças Relacionadas do Hospital Universitário da Universidade Federal do Rio Grande do Norte, Natal, RN, Brasil.

DESCRITORES - Obesidade. Derivação Gástrica. Diabetes Mellitus. Hereditariedade.
RESUMO - Racional - A derivação gastrojejunal em Y-de-Roux é considerada um dos tratamentos mais eficientes para a manutenção de peso a longo prazo; porém, está associada à falhas manifestadas por impossibilidade de manutenção ou reganho de peso e descontrole glicêmico. Objetivo - Estudar os possíveis fatores que influenciam na falha do controle de peso e ou remissão da DM2. Métodos - Estudo do tipo casocontrole, com 159 pacientes submetidos ao bypass gástrico com dois anos ou mais de pós-operatório, sendo selecionados para casos 24 pacientes com perda ponderal insatisfatória e ou ausência de remissão da DM2 e para controle 24 pacientes com perda ponderal satisfatória e ou remissão do DM2, pareados por idade e tempo de pós-operatório. Resultados - Dos 24 casos e 24 controles avaliados, o percentual de reganho de peso foi de $19,32 \%$ e $8,68 \%$ e o percentual de remissão da DM2 foi de $26,6 \%$ e $87,5 \%$ assim como o percentual de recorrência da DM2 foi de $6,6 \%$ e $0,0 \%$ para casos e controles. Observando o IMC máximo pré-operatório, os casos apresentaram em média 53,50 $\pm 12,24 \mathrm{~kg} / \mathrm{m} 2$ e controles $48,77 \pm 5,19 \mathrm{~kg} / \mathrm{m} 2$ sendo que o IMC máximo anterior ao bypass gástrico foi estatisticamente significativo no que se refere à falha no controle de peso ou insucesso da operação. Conclusão Pacientes com IMC máximo inicial elevado ( $\geq 50 \mathrm{~kg} / \mathrm{m} 2)$ apresentaram maior índice de falha na perda ponderal. A intolerância alimentar e diferenças socioeconômicas são consideradas fatores de reganho de peso.

\section{Correspondência:}

Fonte de financiamento: não há Conflito de interesses: não há

Recebido para publicação: 10/11/2012 Aceito para publicação: 31/01/2013

HEADINGS - Obesity. Gastric bypass. Diabetes mellitus. Heredity
ABSTRACT - Background - Roux-en-Y gastric by-pass is considered one of the most effective treatments for maintaining long-term weight loss. However, it is associated to failures manifested by the inability to maintain weight loss, weight gain or poor glycemic control. Objective - Study the possible factors that influence weight loss failure and/or DM2 remission. Methods - Case-control study of 159 patients submitted to gastric by-pass two or more years postoperatively. Twenty-four individuals with unsatisfactory weight loss and/or DM2 remission were selected as cases and 24 with satisfactory weight loss and/or absence of DM2 remission as controls, matched for age and postoperative time. Results - Of the 24 cases and controls evaluated, the percentage weight gain was $19.32 \%$ and $8.68 \%$, percentage DM2 remission $26.6 \%$ and $87.5 \%$ and percentage DM2 recurrence $6.6 \%$ and $0.0 \%$, respectively. Cases and controls exhibited mean maximum preoperative BMI of $53.50 \pm 12.24 \mathrm{~kg} / \mathrm{m} 2$ and $48.77 \pm 5.19 \mathrm{~kg} / \mathrm{m} 2$, respectively. These values were statistically significant in terms of poor weight management or failed surgery. Conclusion - Patients with elevated initial maximum BMI ( $\geq 50 \mathrm{~kg} / \mathrm{m} 2$ ) displayed higher weight loss failure rates. Food intolerance and socioeconomic differences are considered factors in weight gain. 


\section{INTRODUÇÃO}

fisiopatologia da obesidade mórbida é
complexa e não se resume exclusivamente
ao consumo excessivo de calorias. Fatores genéticos, físicos, fisiológicos, psicológicos e comportamentais interagem no processo de ganho de peso. Além disso, o fácil acesso aos alimentos baratos de alto teor calórico, contribui para o ganho ponderal, sobretudo entre as populações de baixa renda ${ }^{23}$.

A obesidade é considerada epidemia mundial e está associada à outras comorbidades, dentre elas doenças cardiovasculares e a diabete melito tipo 2 (DM2). Indivíduos com índice de massa corpórea (IMC) de $35 \mathrm{Kg} / \mathrm{m}^{2}$ têm risco 2,5 vezes maior do que aqueles com IMC entre 20 e $25 \mathrm{~kg} / \mathrm{m}^{2}$, assim como, naqueles com IMC > $40 \mathrm{Kg} / \mathrm{m}^{2}$ esse risco é mais de 10 vezes superior ao dos com IMC normal24.

A obesidade pode predispor ao desenvolvimento da DM2 através da resistência à insulina, intolerância à glicose e falência das células beta pancreáticas, cuja prevalência vem aumentando ao longo dos anos. O IMC é o fator de risco dominante para diabete e ela constitui a principal e mais deletéria comorbidade da obesidade $7,8,11,12,20$.

Com a popularização das operações para obesidade foi possível avaliar os resultados delas sobre os pacientes diabéticos. Ao analisar-se o impacto do tratamento cirúrgico da obesidade sobre a diabete, foi possível constatar que ocorre melhora significativa ou mesmo resolução em longo prazo da doença. Sabese, também, que o impacto da operação sobre DM2 é significativamente maior nos pacientes com menos tempo de doença e uso de menores quantidades de medicamentos, particularmente a insulina ${ }^{19}$.

Em um estudo retrospectivo, realizado na Virgínia, USA, onde foram avaliados 177 pacientes diabéticos e obesos submetidos à derivação gastrojejunal em Y-de-Roux com tempo de pós-operatório superior a cinco anos (máximo de 16 anos) observou-se resolução da DM2 em 89\% nos primeiros cinco anos; porém, o descontrole glicêmico recidivou em $43 \%$ dos operados $^{1,17}$. Em estudo com seguimento de 10 anos no qual foram avaliados pacientes submetidos à derivação gastrojejunal em Y-de-Roux, foi observado que o pico de perda de peso ocorre em torno dos 18-24 meses, período em que foi possível obter percentagem de perda de peso de até $91,8 \%$ em pacientes obesos mórbidos mas não super-obesos) ${ }^{6}$. No entanto, podem ser observados índices de falhas significativos (caracterizada como a manutenção do IMC > $35 \mathrm{~kg} /$ $\mathrm{m}^{2}$ ), afetando sobretudo a população de super-obesos. Em cinco anos, $18 \%$ dos pacientes não mantiveram o IMC abaixo de $35 \mathrm{~kg} / \mathrm{m}^{2}$ (9\% dos obesos mórbidos e $43 \%$ dos super-obesos) enquanto após 10 anos, o índice de falha foi de $35 \%$ (20\% obesos mórbidos e $58 \%$ super-obesos) $)^{6}$.
Dentre os fatores de risco que podem levar ao reganho de peso os principais a serem considerados parecem ser a super-obesidade e o acompanhamento pós-operatório, assim como o comprometimento dos pacientes com as recomendações ${ }^{4,17}$.

Aoestudarainfluênciadosfatoressocioeconômicos após o bypass gástrico no reganho de peso, a literatura observa que não há relação significativa para este fator, apesar de observar dificuldades para pacientes de classes desfavorecidas em adquirir alimentos saudáveis e realizar acompanhamento clínico pós-operatório adequado ${ }^{10,15,26}$.

Este trabalho teve como objetivo estudar a falha na perda ponderal (perda ponderal insuficiente) e/ou a ausência de remissão da DM2 em pacientes submetidos ao bypass gástrico em Y-de-Roux com pelo menos dois anos e verificar a associação entre o insucesso da operação e as variáveis de estudo.

\section{MÉTODOS}

Este estudo foi aprovado pelo Comitê de Ética em Pesquisa da instituição com protocolo 601/11 e foi utilizado termo de consentimento informado. É observacional do tipo caso-controle avaliando 159 pacientes no ano de 2011, de ambos os gêneros, com idade de 20 a 65 anos, submetidos ao bypass gástrico em Y-de-Roux com dois anos ou mais de pós-operatório no Serviço de Cirurgia da Obesidade e Doenças Relacionadas do Hospital Universitário Onofre Lopes da Universidade Federal do Rio Grande do Norte, Natal, RN, Brasil.

Foram selecionados como casos 24 pacientes com perda ponderal insatisfatória (percentual de perda do excesso peso $<50 \%$; IMC atual $>35 \mathrm{Kg} / \mathrm{m}^{2}$, percentual de reganho de peso perdido $\geq 50 \%$ ) e/ou ausência de remissão da DM2 (manutenção do uso de drogas antidiabéticas; HbA1c > 7; glicemia de jejum > 110 $\mathrm{mg} / \mathrm{dl}$; glicemia pós-prandial > $180 \mathrm{mg} / \mathrm{dl}$ ). Como controles, 24 pacientes com perda ponderal satisfatória (percentual de perda do excesso peso > 50\%; IMC atual $<35 \mathrm{Kg} / \mathrm{m}^{2}$; percentual de reganho de peso perdido $\leq 50 \%$ ), e/ou remissão do DM2 (não uso de drogas antidiabéticas; HbA1c < 7, glicemia de jejum < 110 mg/ $\mathrm{dl}$; glicemia pós-prandial $<180 \mathrm{mg} / \mathrm{dl}$ ), pareados por idade e tempo de pós-operatório.

O levantamento foi realizado por meio da análise de prontuários, consulta de enfermagem, entrevista dirigida, avaliação física e laboratorial.

As variáveis estudadas incluíram: idade, tempo de pós-operatório, gênero, procedência (capital ou interior), situação conjugal (com ou sem companheiro), escolaridade, IMC pré-operatório, percentual de remissão da $\mathrm{DM} 2$, presença de $\mathrm{DM} 2$, perda ponderal com cálculo do IMC mínimo (pós-operatório) e após dois anos da operação, cálculo do percentual de reganho do excesso de peso perdido e resultados 
laboratoriais dos exames de rotina pré-operatória ${ }^{21,22}$.

Foi considerada obesidade grave ou grau III o IMC $\geq 40,0$, e super obesos os pacientes com IMC $\geq 50,05$.

A pressão arterial foi aferida com paciente sentado, após 5 min de repouso, com estetoscópio e esfigmomanômetro aneróide para obesos9. Foram considerados hipertensos os que tinham história de doença hipertensiva, os que apresentavam pressão arterial sistólica igual ou superior a $140 \mathrm{mmHg}$ ou diastólica igual ou superior a $90 \mathrm{mmHg}$, e os que estavam em uso de anti-hipertensivos.

Para avaliar o percentual de reganho de peso perdido foram utilizadas as recomendações do Consenso Bariátrico da Sociedade Brasileira de Cirurgia Bariátrica e Metabólica22.

Foram registrados os resultados dos exames laboratoriais pós-operatórios de rotina (glicemia em jejum e pós-prandial, HbA1c, colesterol total, HDL-c, LDL-C, triglicerídeos, insulina basal, $\mathrm{Ht}, \mathrm{Hb}, \mathrm{AST}, \mathrm{ALT}$, ferritina, EAS, vitamina D e vitamina B12).

Segundo a I Diretrizes Brasileiras de Diagnóstico e Tratamento da Síndrome Metabólica, foram considerados portadores de DM2 os pacientes que apresentavam glicemia de jejum de $126 \mathrm{mg} / \mathrm{dl}$ (duas amostras de dias diferentes), ou glicemia de $200 \mathrm{mg} /$ $\mathrm{dl}$ após teste de tolerância oral com $75 \mathrm{~g}$ de glicose, ou já estarem em uso de medicação para tratamento de DM2. A glicemia foi dosada por amostra de sangue, utilizando-se analisador bioquímico ${ }^{21}$.

Para este estudo foram considerados portadores de dislipidemia os pacientes com diagnóstico prévio e que referiram uso de estatinas, anteriormente ao bypass ${ }^{22}$.

Quanto aos sinais relacionados à alimentação, o engasgo foi definido por desconforto à deglutição seguidos ou não de vômito, geralmente relacionados à mastigação e deglutição.

\section{Análise estatística}

Foi realizada análise descritiva das variáveis do estudo verificando sua distribuição relativa e absoluta na forma de tabelas e gráficos. Para verificar a associação entre as variáveis foram utilizados os testes qui-quadrado ou exato de Fisher. Odds ratio foi calculado com intervalos de confiança de $95 \%$ com seus valores ajustados pela regressão logística múltipla (foram selecionadas as variáveis que apresentaram $p$ $<0,25$ ). Para a comparação entre médias foi realizado o teste t-student. A análise estatística foi realizada por meio do programa SPSS versão 17.0, considerando o nível de significância de $5 \%$.

\section{RESULTADOS}

Dos 159 pacientes com dois anos ou mais de seguimento foram selecionados e reavaliados 48 pacientes (24 casos e 24 controles), pareados por idade e tempo de pós-operatório. A idade média em anos foi de $44,58 \pm 11,81$ para casos e $41,38 \pm 9,89$ para controles; o tempo de pós-operatório em meses foi de $42,08 \pm 11,44$ para casos e $42,00 \pm 15,04$ para controles; o IMC inicial (pré-operatório) foi em média 53,50 $\pm 12,24$ $\mathrm{kg} / \mathrm{m} 2$ para casos e $48,77 \pm 5,19 \mathrm{~kg} / \mathrm{m} 2$ para controles; o percentual de reganho do peso perdido foi em média $19,32 \pm 17,76$ para casos e $8,68 \pm 9,84$ para controles e o percentual de ausência de remissão da DM2 foi de $71,43 \%$ para casos e $12,5 \%$ para controles.

Em relação às demais variáveis clinicolaboratoriais, os casos apresentaram, em média, 15,08 $\pm 3,32 \mathrm{mg} / \mathrm{dl}$ de colesterol total a mais que os controles $(p=0,046)$ e a menos, em média, $139,83 \pm 2,33 \mathrm{pg} / \mathrm{ml}$ de vitamina B12 $(p<0,045)$ e $1,24 \pm 0,17 \mathrm{uUI} / \mathrm{ml}$ de insulina basal $(p<0,016)$ (Tabela 1).

TABELA 1 - Média e desvio-padrão dos valores dos exames laboratoriais após dois anos do bypass gástrico para casos e controles

\begin{tabular}{|l|c|c|c|}
\hline Exames laboratoriais & Caso $(\mathbf{n}=24)$ & Controle $(\mathbf{n}=24)$ & $p$ \\
\hline Glicemia de Jejum & $106,25 \pm 49,50$ & $85,2 \pm 9,85$ & 0,052 \\
\hline Glicemia pós-prandial & $122,23 \pm 52,02$ & $104,27 \pm 15,57$ & 0,117 \\
\hline HbAlc & $5,24 \pm 1,04$ & $4,98 \pm 0,70$ & 0,305 \\
\hline Colesterol total & $175,41 \pm 27,04$ & $160,33 \pm 23,72$ & 0,046 \\
\hline HDL & $52,38 \pm 10,45$ & $52,58 \pm 9,16$ & 0,942 \\
\hline Triglicerides & $104,13 \pm 42,12$ & $104,92 \pm 27,73$ & 0,939 \\
\hline Hematócrito & $37,51 \pm 4,65$ & $37,94 \pm 4,07$ & 0,735 \\
\hline Hemoglobina & $12,40 \pm 1,39$ & $12,61 \pm 1,68$ & 0,624 \\
\hline AST/TGO & $25,83 \pm 11,31$ & $27,88 \pm 9,27$ & 0,498 \\
\hline ALT/TGP & $25,71 \pm 12,46$ & $25,96 \pm 7,82$ & 0,934 \\
\hline Ferritina & $59,38 \pm 55,25$ & $102,18 \pm 93,93$ & 0,061 \\
\hline VitD & $31,92 \pm 14,05$ & $32,61 \pm 5,12$ & 0,823 \\
\hline VitB12 & $412,06 \pm 236,73$ & $551,89 \pm 234,40$ & 0,045 \\
\hline Insulina basal & $3,57 \pm 1,64$ & $4,81 \pm 1,81$ & 0,016
\end{tabular}

$p \leq 0,05$ - diferença estatisticamente significativa - teste t-student

Não houve diferença estatisticamente significativa nas porcentagens de pacientes segundo o grupo de estudo (caso ou controle) e nas variáveis gênero, procedência, estado civil e escolaridade (qui-quadrado, $p=0,245 / 0,768$ ) (Tabela 2).

TABELA 2 - Número e porcentagem de pacientes segundo o grupo de estudo e as variáveis sociodemográficas

\begin{tabular}{|c|c|c|c|c|c|c|}
\hline \multirow[t]{2}{*}{ Variáveis } & \multicolumn{2}{|c|}{$\begin{array}{l}\text { Caso } \\
(\mathrm{n}=24)\end{array}$} & \multicolumn{2}{|c|}{$\begin{array}{c}\text { Controle } \\
(n=24)\end{array}$} & \multirow[t]{2}{*}{$p$} & \multirow{2}{*}{$\begin{array}{c}\text { OR } \\
\text { (IC95\%) }\end{array}$} \\
\hline & $\mathrm{N}^{\circ}$. & $\%$ & $\mathrm{~N}^{\circ}$. & $\%$ & & \\
\hline \multicolumn{7}{|l|}{ Sexo } \\
\hline Feminino & 22 & 91,7 & 18 & 75,0 & \multirow{2}{*}{ (1) 0,245} & 3,67 \\
\hline Masculino & 2 & 8,3 & 6 & 25,0 & & $0,55-30,26$ \\
\hline \multicolumn{7}{|l|}{ Procedência } \\
\hline Capital & 16 & 66,7 & 19 & 79,2 & \multirow{2}{*}{ (2) 0,330} & 0,53 \\
\hline Interior & 8 & 33,3 & 5 & 20,8 & & $0,12-2,28$ \\
\hline \multicolumn{7}{|l|}{ Situação conjugal } \\
\hline Com companheiro & 15 & 62,5 & 14 & 58,3 & \multirow{2}{*}{ (2) 0,768} & 1,19 \\
\hline Sem companheiro & 9 & 37,5 & 10 & 41,7 & & $0,32-4,45$ \\
\hline \multicolumn{7}{|l|}{ Escolaridade } \\
\hline Até fundamental & 10 & 41,7 & 9 & 37,5 & \multirow{2}{*}{ (2) 0,768} & 1,19 \\
\hline Média ou superior & 14 & 58,3 & 15 & 62,5 & & $0,62-1,93$ \\
\hline
\end{tabular}

$p \leq 0,05$ - diferença estatisticamente significativa; (1) teste exato de Fisher; (2) Qui-quadrado 
De acordo com a análise de regressão logística (Tabela 3) as variáveis hipertensão arterial sistêmica e antecedentes familiares, DM2 e antecedentes familiares, entalos, gênero e dislipidemia não apresentaram associação estatisticamente significativa com o insucesso do bypass gástrico; por outro lado, o IMC máximo (préoperatório) apresentou associação estatisticamente significativa com o insucesso da operação $(p=0,047)$.

TABELA 3 - Regressão logística múltipla de acordo com as variáveis incluídas $(n=48)$

\begin{tabular}{l|c|c|c|}
\hline Variáveis & OR ajustado & IC 95\% & $p$ \\
\hline Hipertensão arterial sistêmica & 1,29 & $0,17-9,95$ & 0,805 \\
\hline DM 2 & 1,42 & $0,14-14,24$ & 0,767 \\
\hline Entalos & 0,74 & $0,11-4,77$ & 0,748 \\
\hline Antecedente familiar para DM2 & 2,36 & $0,27-20,77$ & 0,438 \\
\hline Gênero & 5,50 & $0,56-54,26$ & 0,145 \\
\hline Dislipidemia & 17,99 & $0,93-347,23$ & 0,056 \\
\hline Antecedente familiar para & 11,87 & $0,95-148,68$ & 0,055 \\
\hline hipertensão & 1,13 & $1,01-1,28$ & 0,047 \\
\hline IMC máximo & \multicolumn{3}{|c|}{0.05 insucesso do bypass gástrico } \\
\hline p $\leq 0,05$-associação estatisticamente significativa com
\end{tabular}

DISCUSSÃO

Ao avaliar os pacientes operados observou-se que alguns não tiveram resultados satisfatórios quanto à perda e manutenção do peso ou controle da DM2. Considerando o reganho de peso, pôde-se observar em outros estudos que ele ocorre em até $63,6 \%$ dos pacientes submetidos à esta operação, variando de acordo com o tempo do pós-operatório e IMC inicial. O acompanhamento pós-operatório é fator importante neste reganho ${ }^{17}$. A falha no controle do peso também está relacionada à super-obesidade 4 .

A ausência de remissão da DM2 pode variar de $11 \%$ a 43\%; a recorrência pode relacionar-se com diversos fatores, dentre eles o tempo de diagnóstico de $\mathrm{DM} 2^{5,25,27}$ e IMCs mais reduzidos ${ }^{9,13}$. Esta pluralidade de resultados mostra a necessidade de aprofundar estudos que possam definir com mais clareza a causa das falhas, visando melhorar a técnica e aumentar o índice de sucesso.

Em relação aos resultados dos exames laboratoriais de rotina verificou-se diferença significativa para casos e controles no que se refere ao colesterol total, vitamina B12 e insulina basal; porém, não se pode afirmar que estes sejam fatores preditivos de falha no tratamento. Metanálise ao avaliar a eficácia da técnica com relação às hiperlipidemias observou que colesterol total em 307 mg.dl após esta operação sofre redução média de $33,2 \mathrm{mg} / \mathrm{dl}^{4}$.

Colabora com estes resultados estudo retrospectivo realizado em Campinas com 386 pacientes nas mesmas condições deste trabalho. Os portadores de DM2 que obtiveram remissão ou não, apresentaram índices semelhantes de colesterol total e insulina basal; também como aqui, observaram insulina basal significativamente menor entre os pacientes com ausência de remissão de DM2 do que nos sem remissão ${ }^{16}$.

Neste estudo, observou-se que a média dos níveis de vitamina B12 estavam maiores que 200 pg/ml, dentro dos parâmetros recomendados ${ }^{18}$; no entanto, achados na literatura mostram carência de vitamina B12, dentre outras, após o bypass possivelmente relacionada às alterações de absorção, baixa qualidade alimentar, redução na ingestão e intolerâncias ${ }^{2,18}$.

Com relação às variáveis sociodemográficas, verificou-se não haver diferença significativa entre casos e controles. Em relação ao gênero, achados semelhantes foram encontrados em metanálises realizadas em 2004 e 2009, com maior frequência de mulheres em $72,6 \%$ e $79,6 \%$, respectivamente ${ }^{3,4}$.

Ao discutir o nível socioeconômico neste estudo, analisado pelo nível de escolaridade, mostrou haver predomínio do nível médio ou superior tanto nos casos quanto nos controles, porém sem diferença significativa. Apoiam-se neste resultado estudos da literatura mostrando não haver diferença entre os resultados de perda de peso e os níveis socioeconômicos ${ }^{1,15,26}$.

Por outro lado, uma pesquisa sobre intolerância alimentar com 47 pacientes submetidos à esta operação realizada em Natal, RN, Brasil, constatou que o nível socioeconômico tem relação significativa com o grau de intolerância alimentar ${ }^{14}$.

Ao fazer a regressão logística, observou-se que o IMC máximo anterior ao bypass foi estatisticamente significativo no que se refere à falha no controle de peso ou insucesso da operação. Este achado já foi apresentado no Canadá, e mostrou que os pacientes com IMC máximo inicial elevado $\left(\geq 50 \mathrm{~kg} / \mathrm{m}^{2}\right)$, apresentaram maior índice de falha na perda ponderal chegando à $58 \%$ dos operados acima de dez anos de acompanhamento 6 .

Por outro lado, em estudo retrospectivo realizado na Universidade de Columbia com 42 pacientes portadores de DM2 após pós-operatório de pelo menos três anos, verificou-se que os pacientes com maior IMC pré-operatório apresentaram melhores resultados na remissão do $\mathrm{DM} 2$, porém o percentual de reganho de peso foi inversamente proporcional para os grupos com e sem remissão do $\mathrm{DM} 2$, respectivamente $37,7 \%$ e $15,4 \%$.

\section{CONCLUSÃO}

O IMC máximo apresentou associação com a falha do bypass gástrico, ou seja, pacientes com peso maior apresentaram mais chances de falhas no controle do peso e da DM2 no pós-operatório. A intolerância alimentar e diferenças socioeconômicas foram consideradas fatores de reganho de peso.

\section{REFERÊNCIAS}

1. ADA. Standards of Medical Care in Diabetes-2010. Diabetes Care. 2010 January 1, 2010;33(Supplement 1):S11-S61.

2. Bloomberg RD, Fleishman A, Nalle JE, Herron DM, Kini $S$. Nutritional deficiencies following bariatric surgery: what have we learned? Obes Surg. 2005 Feb;15(2):145-54. 
3. Buchwald $H$, Avidor $Y$, Braunwald $E$, Jensen MD, Pories W, Fahrbach $\mathrm{K}$, et al. Bariatric surgery: a systematic review and meta-analysis. Jama. 2004 Oct 13;292(14):1724-37.

4. Buchwald $H$, Estok R, Fahrbach K, Banel D, Jensen MD, Pories WJ, et al. Weight and Type 2 Diabetes after Bariatric Surgery: Systematic Review and Meta-analysis. Am J Med. 2009;122(3):248-56.e5.

5. Chikunguwo SM, Wolfe LG, Dodson P, Meador JG, Baugh N, Clore $\mathrm{JN}$, et al. Analysis of factors associated with durable remission of diabetes after Roux-en-Y gastric bypass. Surg Obes Relat Dis. 2010 May-Jun;6(3):254-9.

6. Christou NV, Look D, Maclean LD. Weight gain after short- and long-limb gastric bypass in patients followed for longer than 10 years. Ann Surg. 2006 Nov;244(5):734-40.

7. Colditz GA, Willett WC, Rotnitzky A, Manson JE. Weight Gain as a Risk Factor for Clinical Diabetes Mellitus in Women. Anna Intern Med. 1995 April 1, 1995;122(7):481-6.

8. DeFronzo RA. Lilly lecture 1987. The triumvirate: beta-cell, muscle, liver. A collusion responsible for NIDDM. Diabetes. 1988 Jun;37(6):667-87.

9. DiGiorgi M, Rosen DJ, Choi JJ, Milone L, Schrope B, Olivero-Rivera $L$, et al. Re-emergence of diabetes after gastric bypass in patients with mid- to long-term follow-up. Surg Obes Relat Dis. 2010 Jun;6(3):249-53.

10.Durkin AJ, Bloomston M, Murr MM, Rosemurgy AS. Financial status does not predict weight loss after bariatric surgery. Obes Surg. 1999 Dec;9(6):524-6.

11. Flegal KM, Carroll MD, Kuczmarski RJ, Johnson CL. Overweight and obesity in the United States: prevalence and trends, 1960-1994. International journal of obesity and related metabolic disorders: journal of the International Association for the Study of Obesity. 1998;22(1):39-47.

12. Ford ES, Williamson DF, Liu S. Weight Change and Diabetes Incidence: Findings from a National Cohort of US Adults. Am J Epidemiol. 1997 August 1, 1997;146(3):214-22.

13. Freitas ACT. Cirurgia gastrointestinal no tratamento da diabete tipo 2. ABCD Arq Bras Cir Dig 2007; 20(2):119-26.

14. Godoy CM, Caetano AL, Viana KR, Godoy EP, Barbosa AL, Ferraz EM. Food tolerance in patients submitted to gastric bypass: the importance of using an integrated and interdisciplinary approach. Obes Surg. 2012 Jan;22(1):124-30.
15. Halloran K, Padwal R, Johnson-Stoklossa C, Sharma A, Birch D. Income Status and Approval for Bariatric Surgery in a Publicly Funded Regional Obesity Program. Obes Surg. 2011;21(3):373-8.

16. Hirsch FF, Pareja JC, Geloneze SR, Chaim E, Cazzo E, Geloneze B. Comparison of Metabolic Effects of Surgical-Induced Massive Weight Loss in Patients with Long-Term Remission Versus Nonremission of Type 2 Diabetes. Obes Surg. 2012 Jan 13.

17. Magro DO, Geloneze B, Delfini R, Pareja BC, Callejas F, Pareja JC. Long-term weight regain after gastric bypass: a 5 -year prospective study. Obes Surg. 2008 Jun;18(6):648-51.

18. Malvezzi M, Zago MA. Deficiências de Vitamina B12 e de Folatos: Anemias Megaloblásticas. In: Atheneu E, editor. Hematologia Fundamentos e Prática. São Paulo; 2004. p. 195-209.

19. Pinkney JH, Sjostrom CD, Gale EA. Should surgeons treat diabetes in severely obese people? Lancet. 2001 Apr 28;357(9265):1357-9.

20. Rubin RJ, Altman WM, Mendelson DN. Health care expenditures for people with diabetes mellitus, 1992. The Journal of Clinical Endocrinology and Metabolism. 1994 Apr;78(4):809A-F.

21. SBC. I Diretriz Brasileira de Diagnóstico e Tratamento da Síndrome Metabólica. Arq Bras Cardiol. 2005;84:3-28.

22. SBCBM. Consenso Bariátrico Multissocietário em Cirurgia da Obesidade. Brasil: SBCBM; 2006.

23. Smith BR, Schauer P, Nguyen NT. Surgical Approaches to the Treatment of Obesity: Bariatric Surgery. Endocrinol Metab Clin N Am. 2008;37(4):943-64.

24. Tessier DJ, Eagon JC. Surgical Management of Morbid Obesity. Curr Probl Surg. 2008;45(2):68-137.

25. Varaschim M, Nassif PAN, Moreira LB, Nascimento MM, Vieira GMN, Garcia RF, Sue KM, Cruz MA. Alterações dos parâmetros clínicos e laboratoriais em pacientes obesos com diabetes melito tipo 2 submetidos à derivação gastrojejunal em y de Roux sem anel. Rev Col Bras Cir. 2012; 39(3):178-182.

26. Wallace A, Young-Xu Y, Hartley D, Weeks W. Racial, Socioeconomic, and Rural-Urban Disparities in Obesity-Related Bariatric Surgery. Obes Surg. 2010;20(10):1354-60.

27. Zeve JLM, Tomaz CAB. Cirurgia metabólica - cura para diabete tipo 2. ABCD Arq Bras Cir Dig 2011;24(4): 312-317. 